\title{
Fatores que afetam o aprendizado de alunos do mestrado em Administração em relação à Estatística
}

\author{
Cláudio Damacena* \\ damacena.claudio@gmail.com \\ Martin De La Martinière Petroll \\ martin.petroll@ufsc.br \\ Bruna Silva de Melo \\ brunasmelo@gmail.com
}

*Pontifícia Universidade Católica do Rio Grande do Sul - Porto Alegre, RS / Brasil

http://dx.doi.org/10.1590/1413-2311.02915.58251

Recebido em 01/09/2015

Aprovado em 10/08/2016

Disponibilizado em 31/01/2017

Avaliado pelo sistema "double blind review"

Revista Eletrônica de Administração

Editora-chefe: Aurora Zen

ISSN 1413-2311 (versão "on line")

Editada pela Escola de Administração da Universidade Federal do Rio Grande do Sul.

Periodicidade: Quadrimestral

Sistema requerido: Adobe Acrobat Reader

\section{RESUMO}

A estatística é vista por muitos estudantes como um mal necessário, um obstáculo para a obtenção do tão sonhado diploma. Pesquisas anteriores comprovam que grande parte do desafio do ensino e aprendizagem da estatística é explicado pelas atitudes que os estudantes possuem sobre a disciplina. No Brasil, entretanto, não foram encontrados estudos que ratifiquem tais achados. É nesse sentido, portanto, que se insere o presente trabalho, com o objetivo de analisar os antecedentes do interesse e da ansiedade dos estudantes de Administração em relação à estatística a partir do modelo proposto por Mondéjar-Jiménez e Vargas-Vargas (2010). Além disso, incluiu-se o construto da autoeficácia, que nada mais é do que as crenças que um indivíduo tem sobre suas habilidades para desempenhar um determinado comportamento. A importância da autoeficácia está no fato de que ela pode afetar o interesse e a ansiedade do estudante ao encarar a estatística. A partir de uma abordagem quantitativa, foram coletados dados de 120 alunos de 7 instituições de ensino superior do Sul do Brasil. Os resultados da análise a partir da Modelagem de Equações Estruturais mostram que a percepção da utilidade da estatística para os estudos e para a profissão, e a autoeficácia afetam o interesse e a ansiedade do estudante. Por fim, discutem-se tais achados e sugerem-se táticas de como tornar o processo de ensino e aprendizagem de estatística mais eficaz.

Palavras-Chave: Estatística; Atitude; Interesse; Ansiedade; Utilidade; Autoeficácia. 


\title{
Factors that affect learning towards Statistics according to master of administration students
}

\begin{abstract}
Statistics is seen by many students as a necessary evil, an obstacle to obtain the so desired diploma. Previous research show that much of the challenge of teaching and learning statistics is explained by the attitudes that students have about the subject. In Brazil, however, there are no studies to ratify such findings. Therefore, this study was conducted with the objective of analyzing the antecedents of interest and anxiety of students regarded to statistics, from the model proposed by Mondejar-Jiménez and Vargas-Vargas (2010). Also included is the construct of self-efficacy, which is the beliefs that an individual has on his or her ability to perform a particular behavior. The importance of self-efficacy is the fact that it can affect the interest and anxiety the student has on statistic. Following a quantitative approach, data were collected from 120 management students from seven higher education institutions in southern Brazil. The analysis done with Structural Equation Modeling show that the perception of the statistical utility for studies and for the profession and self-efficacy affect the interest and anxiety of the student. Finally, we discuss these findings and suggest tactics on how to make the process of teaching and learning more effective.
\end{abstract}

Keywords: Statistics; Attitude; Interest; Anxiety; Utility; Self-efficacy 


\title{
Los factores que afectan el aprendizaje de los estudiantes de Maestría en Administración de Empresas en relación a la Estadística.
}

\begin{abstract}
RESUMEN
La Estadística es considerada por muchos estudiantes como un mal necesario, un obstáculo para el título deseado. Gran parte del reto de la enseñanza y del aprendizaje de la Estadística se explica, según estudios previos, por las actitudes que los estudiantes tienen sobre la misma. En Brasil, sin embargo, no fueron encontrados estudios que confirmen estos resultados. El objetivo de esta investigación fue analizar las variables que influencian el interés y la ansiedad de los estudiantes de gestión en relación a Estadística, a partir del modelo propuesto por Mondéjar-Jiménez y Vargas-Vargas (2010). Además, incluye el constructo auto-eficacia, que no es más que la creencia de que una persona tiene sobre su capacidad para realizar un determinado comportamiento. La importancia de la auto-eficacia esta en el hecho de que puede afectar el interés y la ansiedad del estudiante para enfrentar la Estadística. Desde un enfoque cuantitativo, se recogieron datos de 120 estudiantes de siete instituciones de educación superior en el sur de Brasil. Los resultados del examen del modelo de ecuaciones estructurales muestran que la percepción de la utilidad de la Estadística para el estudio y para la profesión, y la auto-eficacia afectan el interés y la ansiedad del estudiante. Finalmente, se discuten estos hallazgos y se indican tácticas para que el proceso de enseñanza y aprendizaje estadístico sea más eficiente.
\end{abstract}

Palabras Clave: Estadística; Actitud; Interés;; Ansiedad; Utilidad; auto-eficacia.

\section{INTRODUÇÃO}

Conforme Junius e Sidell (2009), a estatística é uma disciplina que se preocupa com desenhos experimentais e outras formas de coleta de dados, com a sumarização da informação para auxiliar no seu entendimento, com conclusões a partir dos dados coletados e com a estimação do presente ou prevendo o futuro. Ainda segundo os autores, estatística é uma área fascinante, comparável à investigação conduzida por um detetive.

Sabe-se também que a estatística está associada à matemática (JUNIUS; SIDELL, 2009; BELL, 2008). Nesse sentido, há estudos que medem o conhecimento de estudantes em matemática no mundo. Um deles é o Pisa (Programme for International Student Assessment), uma iniciativa internacional desenvolvido e coordenado pela Organização para Cooperação e Desenvolvimento Econômico (OCDE), cujo objetivo é o de "produzir indicadores que 
contribuam para a discussão da qualidade da educação nos países participantes, de modo a subsidiar políticas de melhoria do ensino básico" (INEP, 2014).

No Brasil, por exemplo, segundo relatório do Pisa de 2012, os estudantes alcançaram indicador abaixo da média dos demais países avaliados pela OCDE, ficando em posição similar a estudantes de países como a Albânia, Jordânia e Tunísia. Comparado aos países da América Latina, o Brasil perde para Chile, México, Uruguai e Costa Rica. Todavia, segundo o mesmo relatório, houve um crescimento desde 2003 no conhecimento dos estudantes em matemática. Portanto, sabe-se, por estudos como o Pisa e outros acadêmicos, que o conhecimento da matemática dos estudantes é específico do seu país de origem (BELL, 2008).

Nesse sentido e devido aos indicadores anteriormente mencionados, espera-se que haja dificuldades, por parte dos estudantes brasileiros, em estatística quando ingressam no Ensino Superior. A maior parte dos cursos de ciências sociais, seja em instituições internacionais, seja no Brasil, possui pelo menos uma disciplina com estatística, aliada ao fato da intensificação da importância da estatística para o entendimento do mundo atual (MONDÉJAR-JIMÉNEZ; VARGAS-VARGAS, 2010).

Estudos anteriores informam que o medo e a ansiedade que parte dos estudantes tem sobre matemática pode comprometer seu desempenho em estatística (JUNIUS; SIDELL, 2009; BELL, 2008). Isso significa que muitos estudantes possuem uma forte aversão à estatística, muitas vezes motivada por preconceito e experiências passadas em cursos de matemática (MONDÉJAR-JIMÉNEZ; VARGAS-VARGAS, 2010). Destarte, níveis altos de ansiedade no estudo de estatística afetam negativamente o aprendizado (ONWUEGBUZIE et al, 2000).

Para tanto, Mondéjar-Jiménez e Vargas-Vargas (2010) sugerem que se deva analisar as motivações e atitudes dos estudantes sobre estatística, detectar os fatores psicológicos (afetivos e cognitivos) que os influenciam com o objetivo de clarificar como tais fatores influenciam a forma com que a estatística é aprendida. Sabe-se, por exemplo, que esses fatores envolvidos na atitude sobre a estatística são multi-dimensionais. Todavia, MondéjarJiménez e Vargas-Vargas (2010) foram os primeiros a pesquisar a estrutura de correlação entre os fatores que determinam as atitudes dos estudantes sobre estatística.

Em resumo, Mondéjar-Jiménez e Vargas-Vargas (2010) encontraram que a utilidade percebida e o interesse reduzem o nível de ansiedade dos estudantes sobre a estatística. Além disso, encontraram diferenças significativas entre estudantes do sexo masculino e do sexo feminino. Ou seja, para os homens, houve um efeito significativo e intenso quando um 
aumento na percepção de utilidade reduzia a ansiedade sobre estatística. Já para as mulheres, a redução na ansiedade foi produzida por um aumento no interesse sobre estatística.

Todavia, uma variável que pouco foi estudada quanto à relação entre ansiedade e os fatores multi-dimensionais sobre estatística é a autoeficácia. Ela é definida como as crenças que um indivíduo tem sobre suas habilidades para desempenhar um determinado comportamento (MACKAY; PARKINSON, 2010). A importância da autoeficácia no estudo da ansiedade em estatística está no fato de que ela prediz a persistência e o esforço de um indivíduo sobre determinada tarefa, onde pessoas com autoeficácia elevada experimentam baixa ansiedade e estão mais dispostas a enxergar as tarefas como não sendo difíceis, mas como sendo desafios que podem ser combatidos (BANDURA, 1994).

Tal construto não foi mensurado por Mondéjar-Jiménez e Vargas-Vargas (2010) e, assim como informado por Mackay e Parkinson (2010), existe literatura sobre o tema, mas baseada em pesquisas norte-americanas e europeias. Portanto, necessita-se estender a pesquisa para incluir perspectivas dos países em desenvolvimento. É nesse sentido, portanto, que se busca, a partir do presente estudo, replicar a pesquisa de Mondéjar-Jiménez e Vargas-Vargas (2010) para a realidade brasileira, incluindo o construto da autoeficácia no modelo.

\section{REFERENCIAL TEÓRICO}

A relação não tão profícua entre estudantes e a estatística é antiga. Segundo Epstein (1987), já naquela época a estatística era encarada por estudantes como se eles fossem a um dentista: os estudantes chegam tensos e ansiosos e apenas se inscrevem na disciplina por ela ser obrigatória e não por terem interesse no assunto (JUNIUS; SIDELL, 2009). Em outras palavras, a estatística é vista, por muitos estudantes, como um mal necessário (UTTL; WHITE; MORIN, 2013).

Todavia, é importante frisar que o desinteresse por estatística pode limitar os estudantes em suas escolhas acadêmicas e profissionais (UTTL; WHITE; MORIN, 2013). Isso porque o conhecimento em estatística é amplamente utilizado em múltiplos campos tanto da Academia como da prática não-acadêmica (CHIOU; WANG; LEE, 2014). Portanto, a aversão à estatística limita os estudantes tanto na decisão do curso a ser cursado na graduação ou na pós-graduação, como na possibilidade de alçar voos maiores em suas carreiras, já que é sabido que muitos empregos com altos salários exigem conhecimento em estatística (UTTL; WHITE; MORIN, 2013). 
Nesse sentido, é necessário que os estudantes entendam a importância de se estudar estatística (CHIOU; WANG; LEE, 2014) e não encará-la como um obstáculo para a obtenção do diploma (LALAYANTS, 2012). Para tanto, é relevante entender os fatores que possam afetar o aprendizado dos estudantes sobre estatística como um diagnóstico para, em seguida, encontrar soluções que desmistifiquem esse campo de estudo.

Existem diversos fatores que podem influenciar no aprendizado de estatística, como o conhecimento fraco em matemática, por exemplo. Porém, professores de estatística se preocupam fortemente em intensificar os aspectos de conteúdo em sala de aula aos alunos ao invés de prestarem atenção aos demais aspectos, como as atitudes dos estudantes em relação à estatística (ZHANG et al., 2012). As atitudes dos estudantes podem tanto ser um obstáculo como uma vantagem para o aprendizado da estatística. Mondéjar-Jiménez e Vargas-Vargas (2010) relatam haver inúmeros estudos que confirmam a correlação entre as atitudes dos estudantes e seus desempenhos em estatística.

Ou seja, as atitudes em relação à estatística podem tanto facilitar como dificultar o aprendizado, influenciando diretamente no entendimento dos conceitos e métodos estatísticos (ONWUEGBUZIE, 2003). As atitudes também podem influenciar os estudantes com relação ao desenvolvimento de habilidades e no uso desse conhecimento estatístico em sua aplicação na carreira profissional (ONWUEGBUZIE, 2000).

Assim sendo, atitudes "não são diretamente observáveis, são aspectos inferidos, consistindo em crenças, sentimentos e predisposições comportamentais sobre um objeto nas quais estão sendo direcionadas" (AUZMENDI, 1992, p. 17, tradução nossa). No presente caso, as atitudes representam a soma de emoções e sentimentos experimentados por um indivíduo durante um certo período de tempo no contexto da aprendizagem à estatística (ZHANG et al., 2012).

Alguns estudos foram realizados para medir e monitorar as atitudes dos estudantes sobre estatística. Para tanto, foram criadas diversas escalas, como a Attitudes Toward Statistics scale (ATS) e a Survey of Attitudes Toward Statistics (SATS), ambas sendo utilizadas em pesquisas mensurando as atitudes de alunos do ensino médio e de graduação (ZHANG et al., 2012).

Todavia e segundo Mondéjar-Jiménez e Vargas-Vargas (2010), existem fatores multidimensionais envolvidos na atitude sobre a estatística, podendo ser tanto cognitivos como afetivos. Devido à sua multidimensionalidade, à discordância de autores quanto ao número de dimensões e seguindo Mondéjar-Jiménez e Vargas-Vargas (2010), o presente 
estudo também utilizará a pesquisa feita por Bayot et al (2005), em que decompõe a atitude em duas subescalas, uma afetiva e a outra avaliativa, ambas bi-dimensionais na sua estrutura.

Seguindo Bayot et al (2005) e Mondéjar-Jiménez e Vargas-Vargas (2010), na subescala afetiva, um fator mede o nível de interesse em estatística e o outro mede o nível de ansiedade dos estudantes quando estudam estatística ou problemas envolvendo estatística. Já a subescala avaliativa consiste também em dois componentes, um medindo a utilidade que os estudantes percebem para os seus estudos correntes e o outro é a utilidade que eles percebem da estatística para suas carreiras profissionais.

Sabe-se, por exemplo, que o nível de interesse em estatística pode afetar a atitude sobre a mesma. Mondéjar-Jiménez e Vargas-Vargas (2010) encontraram que um interesse maior em estatística significativamente reduz a ansiedade dos estudantes à estatística e, consequentemente, aumenta a atitude sobre estatística. Portanto, professores da área devem criar mecanismos que aumentem o interesse dos estudantes sobre estatística, afetando assim, suas atitudes sobre a mesma.

Com relação ao outro fator da escala afetiva do construto da atitude, a ansiedade é uma ativação emocional causada parcialmente por medo de reações fisiológicas adversas a uma situação estressante (HE; FREEMAN, 2010). Tal medo eleva os níveis de ansiedade e emite dúvidas à habilidade de um indivíduo executar o comportamento almejado com sucesso.

No caso da ansiedade à estatística, esta pode ser entendida como sendo a apreensão que ocorre quando um estudante se depara com a estatística em qualquer forma ou nível de dificuldade (ONWUEGBUZIE, 2004), seja cursando a disciplina ou realizando análises estatísticas (CRUISE; CASH; BOLTON, 1985). Segundo Onwuegbuzie e Wilson (2003), cerca de $80 \%$ dos estudantes de pós-graduação experimentam níveis desconfortantes de ansiedade à estatística quando confrontados, por exemplo, com ideias, problemas, questões, situações e avaliações envolvendo a estatística. Tal ansiedade pode provocar desde um fraco desconforto até, em certos casos, resultados mais negativos, como medo, nervosismo e pânico, prejudicando, finalmente, a aprendizagem (ONWUEGBUZIE et al., 1997).

Sabe-se, portanto, que a ansiedade afeta negativamente a aprendizagem de estatística (ZEIDNER, 1991; ELMORE et al., 1993; LALONDE; GARDNER, 1993; ONWUEGBUZIE; SEAMAN, 1995) e, consequentemente, o desempenho na disciplina (ONWUEGBUZIE; SEAMAN, 1995). Isso significa que a ansiedade sobre a estatística está negativamente relacionada às conquistas dos indivíduos sobre o tema (BALOGLU; ZELHART, 2003). 
Segundo Bell (2008), o conhecimento de matemática exerce um papel importante na ansiedade à estatística. Sabe-se que o número de disciplinas completadas envolvendo matemática, se o estudante focou seus estudos em matemática, o intervalo existente entre o último curso de matemática e o momento atual, as habilidades no campo, o uso de calculadoras, entre outros aspectos favorecem ou não no nível de ansiedade, na aprendizagem e no desempenho do estudante quando cursa estatística (ONWUEGBUZIE, 2004). Portanto, a ansiedade à matemática pode predizer o nível de ansiedade à estatística (ONWUEGBUZIE et al., 1997).

A ansiedade depende também do gênero do estudante. $\mathrm{O}$ estudante do sexo feminino, por exemplo, normalmente têm uma ansiedade à estatística maior do que os estudantes do sexo masculino (ONWUEGBUZIE, 1993). Tal resultado pode ser fruto do conhecimento de matemática do passado. Ou seja, e segundo o Pisa (Programme for International Student Assessment) de 2012, no Brasil os estudantes do sexo masculino pontuaram 18 pontos, em média, a mais em matemática do que estudantes do sexo feminino, margem esta estável desde 2003 (INEP, 2014).

Uma explicação possível para a diferença no gênero está, segundo Srite e Karahanna (2006), no fato de que há padrões de comportamento e de expectativas quanto ao papel de cada gênero em determinada sociedade e, normalmente, os indivíduos se comportam conforme o que a sociedade acha apropriado. Ou seja, matemática e estatística, entre outros, teria uma imagem masculina, fazendo com que as estudantes, conscientemente ou não, evitem e tenham um nível de ansiedade maior ao aprender estatística.

Tanto é verdade que, segundo Baloglu (2003), o gênero é um dos antecedentes da ansiedade à estatística. Pertencente aos fatores ambientais, assim como idade e experiências anteriores, o gênero e demais fatores, como os disposicionais - percepção de dificuldade da tarefa e nível de ameaça ao ego -, e os situacionais - fatores imediatos que estão presentes em determinado evento -, todos eles podem levar a um nível de ansiedade que pode comprometer a aprendizagem (ONWUEGBUZIE et al, 2000).

Seguindo com os fatores multidimensionais da atitude de Mondéjar-Jiménez e VargasVargas (2010) e com as escalas de Bayot et al (2005), a subescala avaliativa consiste também em dois componentes, um medindo a utilidade que os estudantes percebem para os seus estudos correntes e o outro é a utilidade que eles percebem da estatística para suas carreiras profissionais. 
Ou seja, os autores supracitados entendem que a atitude à estatística pode ser afetada também pela percepção do estudante sobre se a estatística será útil tanto para os seus estudos como para o seu trabalho profissional. Portanto, é lógico pensar que uma percepção positiva da utilidade da estatística tanto para os estudos como para a profissão afete positivamente a atitude do estudante sobre a estatística. Além disso, Mondéjar-Jiménez e Vargas-Vargas (2010) encontraram que tal percepção positiva reduz os níveis de ansiedade sobre a estatística e que a percepção de utilidade profissional tem um efeito considerável na percepção de utilidade nos estudos de estatística.

Finalmente, Mondéjar-Jiménez e Vargas-Vargas (2010) encontraram diferenças conforme o gênero do estudante no que se refere à percepção de utilidade nos estudos e na ansiedade. Por exemplo, estudantes do sexo masculino, ao perceberem a utilidade da estatística, fez com que houvesse uma redução na ansiedade sobre a mesma. Já para estudantes do sexo feminino, Mondéjar-Jiménez e Vargas-Vargas (2010) encontraram que um aumento no interesse delas sobre estatística reduziu consideravelmente a ansiedade delas sobre a estatística. Por fim, a relação entre utilidade na carreira e nos estudos foi mais intensa nos estudantes do sexo masculino.

Isso significa que o gênero continua sendo uma variável interessante de ser estudada. Tanto é verdade que muitos pesquisadores, segundo He e Freeman (2010), recomendam incorporar características individuais no modelo de pesquisa tanto como variáveis de controle como variáveis independentes para estudos de reações cognitivas, afetivas e ou comportamentais.

Por fim, outro construto interessante de ser estudado é a autoeficácia. Segundo o Mackay e Parkinson (2010), a autoeficácia, popularmente conhecida como autoconfiança, é a crença na habilidade em realizar ações específicas que produzem resultados desejados. Nesse sentido, é de se esperar que indivíduos com autoeficácia elevada tenham ansiedade baixa e vejam as tarefas não como difíceis, mas como desafios que podem ser superados (BANDURA, 1994).

Ainda segundo Mackay e Parkinson (2010), a autoeficácia é um elemento-chave da teoria cognitiva social no campo da aprendizagem e do comportamento individual. A autoficácia é uma percepção e, como tal, pode ser induzida por processos psicológicos ao deliberar sobre informações de múltiplas fontes. Tais fontes podem ser resumidas em quatro categorias: realizações de desempenho, ou o domínio anterior de alguma experiência com um comportamento desejado; experiência vicária ou observações do desempenho dos outros do comportamento desejado; persuasão verbal ou sugestões e comentários dos outros sobre a sua 
capacidade de executar o comportamento desejado; e excitação emocional ou estados fisiológicos causados por situações estressantes (HE; FREEMAN, 2010).

Isso significa que a percepção de autoeficácia ajuda a regular o comportamento de um indivíduo. Destarte, o estudo da ansiedade e da autoeficácia sobre a estatística é interessante. Todavia, pouco ou nada foi encontrado, até o presente momento, de estudos que buscassem incluir a autoeficácia com as atitudes dos estudantes sobre a estatística. Os autores do presente estudo entendem que tal relação seja importante para o aprofundamento do conhecimento sobre o assunto. E, conforme Bandura (1994), entende-se que a autoeficácia seja antecedente do interesse por estatística e da ansiedade. Ou seja, um estudante que tenha confiança em si próprio terá uma probabilidade maior de enxergar a estatística como sendo um desafio, aumentando, consequentemente, o interesse pelo tema. Ao mesmo tempo, é lógico entender que um indivíduo autoconfiante tenha um nível de ansiedade menor do que um indivíduo pouco ou nada autoconfiante em relação, neste caso, à estatística.

Portanto, estabelecem-se as seguintes hipóteses:

Hla: A percepção da utilidade da estatística na carreira profissional influencia positivamente o interesse na disciplina.

H1b: A percepção da utilidade da estatística na carreira profissional influencia positivamente a percepção da utilidade da estatística nos estudos.

H2a: A percepção da utilidade da estatística nos estudos influencia negativamente a ansiedade à estatística.

H2b: A percepção da utilidade da estatística nos estudos influencia positivamente o interesse na disciplina.

H3: O nível de interesse em estatística tem um efeito negativo sobre o nível de ansiedade à estatística.

H4a: A autoeficácia influencia positivamente o interesse na disciplina.

H4b: A autoeficácia influencia negativamente a ansiedade à estatística.

H5a: O grupo feminino da amostra apresenta um nível de ansiedade à estatística maior que o grupo masculino.

H5b: Os efeitos testados nas hipóteses Hla a H4b são diferentes para os grupos masculino e feminino. 
Finalmente, além de replicar o estudo de Mondéjar-Jiménez e Vargas-Vargas (2010), conforme as hipóteses H1a, H1b, H2a, H2b e H3, criou-se as H4a, H4b e as H5a e H5b como extensão ao que já foi estudado até então, principalmente as $\mathrm{H} 4$, com a inclusão da autoeficácia no modelo, enquanto que as H5 são frutos do que foi comentado anteriormente no referencial teórico e que também, em certo grau, foi estudado por alguns dos autores citados na presente seção.

É importante ainda frisar que, apesar de ainda haver um preconceito quanto a estudos replicados, Easley, Madden e Dunn (2000) afirmam que pesquisas desse gênero são inquestionáveis e críticas para o avanço da ciência, pois auxiliam no desenvolvimento e no refinamento das teorias científicas, sem esquecer na potencialização da generalização dos resultados. Por tudo isso, entende-se que a presente pesquisa, além de almejar a generalização dos resultados para outra realidade que não a europeia, também inclui outro construto que poderá servir de base para futuros estudos com o intuito máximo de entender, cada vez mais, como educadores, professores e demais profissionais da área, podem exercer o seu papel para desmistificar o estudo da estatística aos estudantes e, finalmente, incentivá-los a apreciar este campo de estudo tão importante tanto para a academia como para a vida profissional.

\section{PROCEDIMENTOS METODOLÓGICOS}

O presente estudo seguiu uma abordagem quantitativa e descritiva. A coleta de dados foi realizada com 120 alunos de 7 instituições de ensino superior do Sul do Brasil, entre março e abril de 2014, através de um questionário online. O link do questionário foi enviado aos coordenadores dos Cursos de Mestrado em Administração, com a solicitação que o mesmo fosse enviado aos alunos. Todos os alunos que participaram da pesquisa estavam cursando o mestrado em Administração. A escolha por alunos de pós-graduação se deve ao fato de haver, até o momento, poucos estudos que focam em estudantes de pós-graduação, ao contrário de pesquisas envolvendo estudantes de graduação (LALAYANTS, 2012). Ressalta-se, ainda, que foi feito um teste de médias entre as amostras das universidades e os resultados mostraram não haver diferenças significativas nas respostas.

Conforme o referencial teórico, cinco construtos foram avaliados: 1) ansiedade em relação à estatística; 2) utilidade da estatística nos estudos; 3) utilidade da estatística no trabalho (profissional); 4) interesse em estatística; e 5) autoeficácia, conforme a Tabela 1. As escalas utilizadas para medir os construtos relacionados com a Estatística foram traduzidas e 
adaptadas de Mondéjar-Jiménez e Vargas-Vargas (2010), utilizando a técnica de back translation (BRISLIN, 1976; DOUGLAS; CRAIG, 2007). Já a escala utilizada para medir a autoeficácia também foi traduzida e adaptada de Chen, Gully e Eden (2001) com a mesma técnica.

Para fins de comparação com o estudo realizado por Mondéjar-Jiménez e Vargas-Vargas (2010) e considerando as hipóteses propostas, a análise foi realizada separadamente para o grupo formado por homens e pelo grupo formado por mulheres. As hipóteses foram testadas através da técnica de Modelagem de Equações Estruturais. Para tanto, o software utilizado foi o SmartPLS 2.0, recomendado, principalmente, quando o tamanho da mostra é pequeno e não apresenta normalidade (HAIR et al., 2014).

\section{RESULTADOS}

A seção está organizada em três blocos, inicialmente o modelo de mensuração é avaliado. Logo após, o modelo estrutural e analisado e as hipóteses testadas. Por último, a análise da invariância dos modelos formados pelos grupos de alunos do gênero masculino e do feminino.

\subsection{Avaliação do modelo de mensuração}

$\mathrm{Na}$ avaliação preliminar do modelo foi verificado que alguns itens estavam comprometendo o modelo, no que se refere à validade convergente, discriminante ou na confiabilidade da consistência interna. Decidiu-se pela exclusão de sete (07) itens que apresentam carga muito baixa $(<0,6)$ em seu construto. Esses itens excluídos são apresentados na Tabela 1, sombreados. Como se pode notar, todas as cargas dos itens são superiores a 0,6, a mais baixa é a AE1, primeiro item do construto autoeficácia. Também pode ser observada que a variância média extraída (AVE de Average Variance Extracted) de cada construto foi superior a 0,5. Esses números ratificam a validade convergente do modelo (HAIR et al., 2014). Os alfas de Cronbach e os indicadores da confiabilidade composta (CC) atestam a confiabilidade da consistência interna de todos os construtos, pois seus valores são superiores a 0,7 (HAIR et al., 2014).

Tabela 1 - Construtos e seus respectivos itens

\begin{tabular}{crrrrr}
\hline Código & Construtos e itens & Cargas & Cronbach & CC & AVE \\
\hline Ansiedade & & 0,85 & 0,88 & 0,53 \\
\cline { 3 - 5 }
\end{tabular}

REAd | Porto Alegre - Edição 85 - N 3 - Setembro / Dezembro 2016 - p. 413 - 434 
Item01 Não sou muito bom em estatística.

Item07 Fico calmo e relaxado diante de um problema de estatística.

Item09 Fico muito nervoso quando trabalho com estatística.

Item12 Quando estou diante de um problema de estatística eu não consigo nensar com clareza

Item21 Pensar em seguir um curso de estatística me deixa chateado.

Item22 Tenho medo de estatística porque considero um dos temas mais detestáveis.

Item23 Tenho confiança em mim diante de um problema estatístico.

0,800

\section{AE1 Sou capaz de atingir a maioria das metas que estabeleço para mim $\quad 0,609$}

AE2 Ao me deparar com tarefas difíceis, tenho certeza de que vou $\quad 0,786$ realizá-las até o final.

AE3 Em geral, acredito que posso obter os resultados que são importantes $\quad 0,663$ vara mim.

AE4 Acredito que posso ter o máximo de sucesso em qualquer esforço no $\quad 0,654$ qual utilize a minha mente.

AE5 Serei capaz de superar com êxito muitos desafios. $\quad 0,834$

AE6 Estou confiante de que posso realizar, de forma eficaz, muitas $\quad 0,786$ tarefas diferentes.

AE7 Posso fazer a maioria das tarefas muito bem, em comparação com $\quad 0,721$ outras pessoas.

AE8 Posso realizar as tarefas muito bem, mesmo quando as coisas estão $\quad 0,797$

\begin{tabular}{|c|c|c|c|c|c|}
\hline & Interesse pela Estatística & & \multirow{7}{*}{0,82} & \multirow{7}{*}{0,88} & \\
\hline Item13 & $\begin{array}{l}\text { Fico muito entusiasmado com a possibilidade de utilizar estatística } \\
\text { em meu trabalho }\end{array}$ & 0,857 & & & \\
\hline Item17 & $\begin{array}{l}\text { Gostaria de continuar a minha formação em estatística fazendo } \\
\text { cursos avançados. }\end{array}$ & 0,786 & & & 0,66 \\
\hline Item18 & $\begin{array}{l}\text { A maioria das pessoas se beneficiaria se fizesse um curso de } \\
\text { estatística. }\end{array}$ & 0,767 & & & \\
\hline Item 24 & Estatística é agradável e desafiante para mim. & \multirow[t]{3}{*}{0,836} & & & \\
\hline Item 14 & $\begin{array}{l}\text { Se eu tivesse a chance gostaria de me inscrever em cursos de } \\
\text { estatística, embora não exista a necessidade. }\end{array}$ & & & & \\
\hline Item15 & Eu uso a estatística para me divertir. & & & & \\
\hline
\end{tabular}

Item15 Eu uso a estatística para me divertir.

\section{Utilidade na carreira}

Item02 Acredito que a estatística será uma ferramenta útil na minha 0,855 profissão.

Item04 O conhecimento de estatística aumentaria minhas oportunidades de $\quad 0,823$ emprego.

Item11 Treinamento em estatística poderia melhorar a minha experiência profissional.

Item26 Treinamentos em estatística são importantes para o meu $\quad 0,836$ desenvolvimento na minha área de estudo.

Item27 Acho que seria importante exigir estatística no treinamento para uma $\quad 0,775$ profissão.

Item06 Um treinamento em estatística me forneceria uma melhor compreensão das pesquisas sobre o meu campo de estudo.

Item08 Acho que há assuntos mais importantes do que estatística para o desenvolvimento profissional.

Item19 O conteúdo de estatística não é muito interessante na maioria dos casos.

\section{Utilidade nos estudos}

Item05 Um bom pesquisador deve ter estudado estatística. $\quad 0,652$

Item16 Estudar estatística é perda de tempo. $\quad 0,679$

Item20 A pesquisa científica requer a estatística como uma ferramenta $\quad 0,758 \quad 0,72 \quad 0,82 \quad 0,54$ imprescindível.

Item25 Estatísticas são matematicamente úteis no meu desenvolvimento (futuro).

Item03 Seria melhor deixar a análise estatística para "especialistas" e não 
incluí-la como uma tarefa para os profissionais que nada sabem

sobre $\mathrm{o}$ assunto.

Item10 Estatística pode ser uma ferramenta útil nas pesquisas científicas, mas não para o profissional comum.

Fonte: Dados provenientes da presente pesquisa

Os dados da Tabela 2 permitem concluir que o modelo apresenta validade discriminante. Na linha diagonal da Tabela 2 são apresentadas as raízes quadradas das AVE’s de cada um dos construtos. Esses valores devem ser superiores às correlações entre os construtos (FORNELL; LARCKER, 1981). Por exemplo, a AVE do construto é 0,53. A sua raiz quadrada é igual e 0,73 e, como se vê na Tabela 2, esse valor é superior à correlação da Ansiedade com autoeficácia $(-0,405)$, com Interesse $(-0,646)$, Utilidade na carreira $(-0,368)$ e, finalmente, com Utilidade nos estudos $(-0,401)$.

Tabela 2 - Validade discriminante: correlações x AVE

\begin{tabular}{|c|c|c|c|c|c|}
\hline & Ansiedade & Autoeficácia & Interesse & Util. carreira & Util. estudos \\
\hline Ansiedade & 0,730 & & & & \\
\hline Autoeficácia & $-0,405$ & $\mathbf{0 , 7 3 5}$ & & & \\
\hline Interesse & $-0,646$ & 0,358 & $\mathbf{0 , 8 1 2}$ & & \\
\hline Util. carreira & $-0,368$ & 0,194 & 0,772 & $\mathbf{0 , 8 0 8}$ & \\
\hline Util. estudos & $-0,401$ & 0,329 & 0,705 & 0,719 & $\mathbf{0 , 7 3 8}$ \\
\hline
\end{tabular}

Fonte: Dados provenientes da presente pesquisa

\subsection{Análise do modelo estrutural e teste de hipóteses}

Os resultados apresentados na Tabela 3 mostram que apenas uma hipótese não foi aceita, ou seja, a H2a que afirma que a percepção da utilidade da estatística nos estudos influencia negativamente a ansiedade à estatística. Os resultados indicam, portanto, que as variáveis "interesse na disciplina", e "autoeficácia" influenciam negativamente e de forma significa a ansiedade que os alunos sentem em relação à estatística. $\mathrm{O}$ tamanho do efeito da variável “interesse na disciplina" sobre a ansiedade à estatística pode ser considerado grande. Os dados da Tabela 3 também explicitam que o interesse pela disciplina é fortemente influenciado pela percepção da utilidade da estatística no trabalho ou na carreira. De forma moderada as variáveis "utilidade no estudo" e a "autoeficácia” influenciam o interesse pela disciplina.

Tabela 3 - Resultados dos testes de hipóteses 


\begin{tabular}{|c|c|c|c|c|}
\hline & Hipóteses & $\beta$ & $\begin{array}{l}\text { Desvio } \\
\text { Padrão }\end{array}$ & t-value \\
\hline $\mathrm{H}_{1 \mathrm{a}}$ & Util. carreira -> Interesse & 0,564 & 0,073 & $7,743 * *$ \\
\hline $\mathrm{H}_{1 b}$ & Util. carreira -> Util. estudo & 0,719 & 0,042 & $16,990 * *$ \\
\hline $\mathrm{H}_{2 \mathrm{a}}$ & Util. estudo -> Ansiedade & 0,141 & 0,101 & 1,392 \\
\hline $\mathrm{H}_{2 b}$ & Util. estudo -> Interesse & 0,244 & 0,076 & $3,195^{* *}$ \\
\hline $\mathrm{H}_{3}$ & Interesse -> Ansiedade & $-0,670$ & 0,090 & $7,459 * *$ \\
\hline $\mathrm{H}_{4 \mathrm{a}}$ & Autoeficácia -> Interesse & 0,168 & 0,060 & $2,815^{* *}$ \\
\hline $\mathrm{H}_{4 b}$ & Autoeficácia -> Ansiedade & $-0,212$ & 0,084 & $2,520 *$ \\
\hline
\end{tabular}

Fonte: Dados provenientes da presente pesquisa

Para a verificação da hipótese H5a, foi realizado um teste de médias. Inicialmente, foi criada uma nova variável a partir das médias dos itens que formam o construto ansiedade. Logo após, utilizando essa nova variável, procedeu-se o teste de médias entre os grupos formados pelas alunas e alunos da amostra pesquisada. Os resultados do teste podem ser visualizados na Tabela 4. Nota-se, portanto, que a hipótese H5a foi confirmada $(t=3,73$, $\mathrm{p}<0,000)$, ou seja, as mulheres $(\mathrm{m}=3,77)$ da amostra apresentaram um nível de ansiedade maior do que os homens $(\mathrm{m}=2,94)$ sobre estatística.

Tabela 4 - Teste de médias: gênero x ansiedade

\begin{tabular}{llllll}
\hline & N. & Média & Desvio padrão & t-test & Sig. \\
\hline Homens & 71 & 2,94 & 1,18 & \multirow{2}{*}{3,733} & \multirow{2}{*}{0,000} \\
Mulheres & 49 & 3,77 & 1,23 & & \\
\hline
\end{tabular}

Fonte: Dados provenientes da presente pesquisa

Finalmente, a última hipótese do estudo, H5b, envolve uma análise de dois modelos, um para o grupo formado pelos homens e outro pelas mulheres. Essa hipótese prevê que os efeitos testados nas hipóteses H1a a H4b apresentam diferenças entre os grupos. Os resultados da análise estão apresentados nas Tabelas 5 e 6 . Os dados da Tabela 5 indicam os testes de hipóteses por grupo. Como se pode notar, há duas importantes diferenças: 1) os efeitos da percepção de utilidade da estatística no estudo sobre o interesse na disciplina somente são significativos no grupo masculino $(\mathrm{t}=4,873, \mathrm{p}<0,000)$; e 2) os efeitos da autoeficácia sobre o interesse na disciplina são significativos somente no grupo feminino.

Tabela 5 - Resultados do teste da hipótese $\mathrm{H}_{5 b}$

\begin{tabular}{cc}
\hline Homens & Mulheres \\
\hline
\end{tabular}




\begin{tabular}{lcccccc} 
& $\beta$ & $\begin{array}{c}\text { Desvio } \\
\text { Padrão }\end{array}$ & t-value & $\beta$ & $\begin{array}{c}\text { Desvio } \\
\text { Padrão }\end{array}$ & $t$-value \\
\hline Util. carreira -> Interesse & 0,419 & 0,102 & $4,119^{* *}$ & 0,708 & 0,081 & $12,664^{* *}$ \\
Util. carreira -> Util. estudo & 0,740 & 0,055 & $13,498^{* *}$ & 0,715 & 0,056 & $12,664^{* *}$ \\
Util. estudo -> Ansiedade & $-0,018$ & 0,202 & 0,091 & 0,140 & 0,133 & 1,053 \\
Util. estudo -> Interesse & 0,453 & 0,093 & $4,873^{* *}$ & 0,004 & 0,113 & 0,036 \\
Interesse -> Ansiedade & $-0,492$ & 0,162 & $3,036^{* *}$ & $-0,726$ & 0,113 & $13,8326^{* *}$ \\
Autoeficácia -> Interesse & 0,099 & 0,059 & 1,669 & 0,282 & 0,099 & $2,845^{* *}$ \\
Autoeficácia -> Ansiedade & $-0,257$ & 0,130 & $1,972^{*}$ & $-0,214$ & 0,124 & 1,716 \\
\hline
\end{tabular}

Fonte: Dados provenientes da presente pesquisa

A Tabela 6 permite visualizar os resultados do teste de diferenças dos efeitos entre os dois grupos analisados. Percebe-se, então, que duas relações apresentam diferenças significativas entre homens e mulheres: 1) a relação entre a percepção da utilidade da estatística na carreira e o interesse pela disciplina é muito maior entre as mulheres $(\mathrm{h}=0,419 \times \mathrm{m}=0,708)$; e 2$)$ a percepção de utilidade da estatística no estudo e o interesse pela disciplina é bem maior entre os homens $(\mathrm{h}=0,453 \times \mathrm{m}=0,004)$.

Tabela 6 - Comparação de coeficientes entre os sexos.

\begin{tabular}{lccc}
\hline & $\begin{array}{c}\beta \text { Diferença entre } \\
\text { homens e mulheres }\end{array}$ & $\begin{array}{l}t \text {-value } \\
\end{array}$ & Sig. \\
\hline Util. carreira -> Interesse & $\mathbf{0 , 2 7 2}$ & $\mathbf{2 , 2 3 7}$ & $\mathbf{0 , 0 2 7}$ \\
Util. carreira -> Util. estudo & 0,025 & 0,311 & 0,756 \\
Util. estudo -> Ansiedade & $-0,158$ & 0,659 & 0,512 \\
Util. estudo -> Interesse & $\mathbf{0 , 4 4 9}$ & $\mathbf{3 , 0 9 9}$ & $\mathbf{0 , 0 0 2}$ \\
Interesse -> Ansiedade & 0,234 & 1,194 & 0,235 \\
Autoeficácia -> Interesse & $-0,183$ & 1,700 & 0,092 \\
Autoeficácia -> Ansiedade & $-0,043$ & 0,265 & 0,791 \\
\hline
\end{tabular}

Fonte: Dados provenientes da presente pesquisa

Encerrando os resultados, frisa-se que o presente estudo encontrou interessantes achados, uns corroborando com pesquisas anteriores, e outros inéditos sobre a influência da estatística nos estudantes brasileiros, os quais serão destacados a seguir.

\section{CONSIDERAÇÕES FINAIS}

A estatística não deve ser encarada pelos estudantes como um obstáculo para obter o diploma (CHIOU; WANG; LEE, 2014), mas sim como uma aliada em sua vida profissional, 
já que é sabido que muitos empregos com altos salários exigem conhecimento em estatística (UTTL; WHITE; MORIN, 2013). Além disso, o conhecimento em estatística permite que se haja um entendimento do mundo atual (MONDÉJAR-JIMÉNEZ; VARGAS-VARGAS, 2010).

Nesse sentido, é relevante entender os fatores que possam afetar o aprendizado dos estudantes sobre estatística como um diagnóstico para, em seguida, encontrar soluções que desmistifiquem esse campo de estudo. Com base em diversos estudos e, principalmente, a partir dos achados de Mondéjar-Jiménez e Vargas-Vargas (2010), a presente pesquisa buscou diagnosticar os fatores que afetam o aprendizado em estatística por estudantes de pósgraduação de 7 universidades do Sul do Brasil.

Para tanto, e novamente corroborado por Mondéjar-Jiménez e Vargas-Vargas (2010), analisou-se as atitudes dos estudantes sobre estatística tanto sob aspectos afetivos como cognitivos. Com relação aos afetivos, optou-se por acompanhar Bayot et al (2005) e Mondéjar-Jiménez e Vargas-Vargas (2010) ao medir os fatores do nível de interesse em estatística e também o nível de ansiedade dos estudantes com relação à estatística. Já quanto aos cognitivos, mediu-se as percepções dos estudantes sobre a utilidade da estatística para seus estudos correntes e também para suas carreiras profissionais (BAYOT et al., 2005; MONDÉJAR-JIMÉNEZ; VARGAS-VARGAS, 2010).

Portanto, a intenção inicial do presente estudo é o de replicar os achados de MondéjarJiménez e Vargas-Vargas (2010) para a realidade brasileira, resultando no avanço, mesmo que limitado, da ciência ao auxiliá-la no desenvolvimento e no refinamento de teorias e na potencialização da generalização dos resultados (EASLEY; MADDEN; DUNN, 2000).

Todavia, o presente estudo buscou algo mais e, neste caso, incluiu outro construto que pode servir de base para futuros estudos com o intuito máximo de entender, cada vez mais, como educadores, professores e demais profissionais da área, podem exercer o seu papel para desmistificar o estudo da estatística aos estudantes e, finalmente, incentivá-los a apreciar este campo de estudo tão importante tanto para a academia como para a vida profissional. $\mathrm{O}$ construto chama-se autoeficácia.

Com tudo isso e após a análise dos dados com auxílio da técnica de Modelagem de Equações Estruturais, alguns achados interessantes foram encontrados. Inicialmente, e ao contrário do que foi encontrado por Mondéjar-Jiménez e Vargas-Vargas (2010), a percepção de utilidade por parte dos estudantes participantes da amostra quanto à estatística nos seus estudos afetou positivamente o interesse na disciplia, mas não a ansiedade a ela. Isso significa 
que o estudante entende que a estatística, sendo útil para seus estudos acadêmicos, aumenta o seu interesse por ela, mas a percepção de utilidade nos estudos não diminui a sua ansiedade ao enfrentá-la nos seus estudos. Tais efeitos podem denotar que, mesmo sendo útil e de interesse do estudante, a estatística, se levado em consideração a H2a, não afeta significativamente e negativamente o nível de ansiedade do estudante.

Corroborado com Mondéjar-Jiménez e Vargas-Vargas (2010), a presente pesquisa encontrou que a percepção de utilidade da estatística na carreira profissional fez com que aumentasse o interesse em estatística. Nesse sentido, segue-se em dizer que os estudantes entendem a importância da estatística em sua vida profissional, o que é salutar, já que eles foram ou são estudantes de pós-graduação em Administração, profissão esta que necessita, de seus profissionais, professores ou pesquisadores, conhecimentos em matemática e, claro, em estatística.

Outro achado interessante e que corrobora com o estudo de Mondéjar-Jiménez e VargasVargas (2010) é de que o nível de interesse do estudante por estatística afeta o seu nível de ansiedade quanto à mesma. Ou seja, quanto mais interessado o estudante estiver sobre o assunto, menos ansioso estará ao cursá-la. Nota-se, portanto, a importância de se incentivar o interesse do aluno por estatística, pois isso acarretará em menos ansiedade e, consequentemente, em mais aprendizagem sobre a disciplina.

Para tanto, alguns estudos sugerem que professores de disciplinas envolvendo estatística utilizem algumas técnicas, como: a) o uso do humor (SCHACHT; STEWART, 1990); b) o uso de aprendizagem colaborativa (DOLINSKY, 2001); c) o uso da estatística em situações práticas e do cotidiano dos estudantes (PAN; TANG, 2004); d) o uso do computador aliado a aplicações do mundo real (FORTE, 1995); entre outras.

Ou seja, os estudantes não devem enxergar a estatística como um mal necessário (UTTL; WHITE; MORIN, 2013) ou ainda ir às aulas sobre o assunto com o mesmo comportamento de como se fossem a um dentista, onde tensão, murmúrios, olhos revirados, suor e desistência podem dificultar ainda mais a aprendizagem à estatística (EPSTEIN, 1987). Mesmo sendo uma afirmação de quase 30 anos atrás, nota-se, pela experiência prática ou por estudos anteriores, que muitas ou todas as sugestões anteriormente elencadas continuam não fazendo parte da conduta de muitos professores da área (JUNIUS; SIDELL, 2009).

O presente estudo também encontrou, assim como em Mondéjar-Jiménez e Vargas-Vargas (2010), que a percepção de utilidade da estatística na carreira profissional de um indivíduo afeta positivamente a percepção do mesmo sobre a utilidade do seu estudo. Isso significa que 
o entendimento de que a estatística é ou será útil para a sua vida profissional faz com que o estudante entenda que a estatística é útil também para os seus estudos. Tal resultado é lógico, pois e ao contrário do que muito se ouve nos corredores das universidades em relação a diversas disciplinas, a estatística pode ser colocada em prática, transpondo o seu conteúdo teórico do mundo acadêmico para a realidade profissional. Portanto, o resultado demonstra que os estudantes entendem a importância da estatística em suas vidas. O que falta, talvez, é aumentar o interesse e diminuir a ansiedade a partir da desmistificação do tema e o uso de outras formas de ensino, conforme citado anteriormente.

Em seguida, esta pesquisa inseriu o construto da autoeficácia que, segundo Mackay e Parkinson (2010), é a crença na habilidade em realizar ações específicas que produzem resultados desejados. Nesse sentido, entende-se que a autoeficácia ou autoconfiança pudesse predizer o nível de interesse e o de ansiedade. Ou seja, esperava-se que indivíduos com autoeficácia elevada tivessem ansiedade baixa e vissem as tarefas não como difíceis, mas como desafios que pudessem ser superados (BANDURA, 1994). E foi o que a presente pesquisa encontrou: a autoeficácia influencia positivamente o interesse por estatística e negativamente a ansiedade à estatística.

Isso significa que um estudante, no presente caso, que se sinta autoconfiante faz com que aumente o interesse por determinado assunto e diminua o nível de ansiedade para realizar determinada tarefa envolvendo o mesmo assunto. Portanto, a percepção de autoeficácia ajuda a regular o comportamento de um indivíduo.

Nesse sentido, é salutar que professores de estatística, além de incentivarem o interesse dos estudantes por estatística a partir de tudo o que já foi abordado anteriormente, também busquem diagnosticar o nível de autoeficácia de seus alunos. Com isso, é possível desenvolver a auto-eficácia a partir do incentivo e do acompanhamento ao aluno no seu domínio em tarefas difíceis que possam ser úteis também em sua vida profissional e também em mostrar a eles outros que conquistaram desempenhos excelentes a partir de um comportamento autoconfiante (BANDURA, 1994).

Para encerrar a discussão dos resultados, a presente pesquisa ratificou que há diferenças nos efeitos dos construtos avaliados conforme o gênero do estudante. Isso significa que os estudantes do sexo masculino e os do sexo feminino não, necessariamente, percebem a estatística ou são afetados por ela de maneira similar. Muito pelo contrário. Inicialmente, encontrou-se que as estudantes da amostra apresentavam um nível de ansiedade à estatística maior do que os estudantes do sexo masculino, corroborado por Onwuegbuzie (1993). 
Novamente, esse resultado pode ser um efeito tanto do conhecimento de matemática, cuja diferença entre homens e mulheres é ratificado por pesquisas mundialmente reconhecidas como o Pisa (Programme for International Student Assessment) aliado a padrões de comportamento e de expectativas quanto ao papel de cada gênero em determinada sociedade.

Portanto, era de se esperar que as mulheres tivessem uma ansiedade maior do que os homens no estudo de estatística. Além disso, outro achado é interessante de ser mencionado: estudantes do sexo feminino, ao perceberem a utilidade da estatística em suas carreiras atuais ou futuras, interessam-se muito mais pela disciplina do que os estudantes do sexo masculino. Em contrapartida, os homens, ao perceberem a utilidade da estatística nos estudos, interessam-se muito mais pela disciplina do que as mulheres. Isso significa que homens e mulheres podem ser estimuladas por professores de estatística de maneiras diferentes: o professor deve mostrar a importância da estatística tanto para os estudos na disciplina como para a vida profissional de seus alunos. Somente dessa maneira conseguirão despertar o interesse de todos os estudantes na área.

Conclui-se que o presente estudo foi importante no sentido de replicar os achados de Mondéjar-Jiménez e Vargas-Vargas (2010) para a realidade brasileira, mas também em acrescentar a autoeficácia como um construto relevante para entender como o comportamento dos estudantes pode ser diagnosticado e modificado para que a estatística seja vista, em um futuro próximo, como uma área do conhecimento necessária e agradável tanto academicamente como na vida profissional. Para que isso aconteça, é importante que professores e alunos se esmerem em ensinar e em aprender de maneira aprazível e reduzindo a ansiedade. Além disso, o estudante será autoeficaz e terá interesse sobre o tema se ele for estimulado a entender que a estatística é útil não somente para conseguir um diploma, mas também para a sua carreira profissional.

Finalmente, é imperativo informar que a pesquisa aqui descrita apresenta limitações. Inicialmente, os resultados da mesma não podem ser generalizados devido ao tipo de amostragem - não probabilística e por julgamento - escolhida (MALHOTRA, 1999). Além disso, a escolha do perfil dos participantes também é uma limitação. Ou seja, talvez os resultados fossem outros se fossem abordados estudantes de pós-graduação de cursos outros, como Filosofia ou História ou ainda de Engenharias e de Matemática, por exemplo.

Portanto, os resultados devem ser vistos com ressalvas, mas também com base para novos estudos. Sugerem-se, assim, pesquisas que ampliem o estudo do comportamento dos estudantes sobre estatística, desde enfatizando as atitudes e suas variáveis, como foi feito 
aqui, como a autoeficácia e seus desdobramentos para o ensino da estatística. Outras pesquisas podem ser feitas com novas amostras, probabilísticas ou não, e comparando diversos cursos como aqueles informados anteriormente. Além disso, uma pesquisa mais aprofundada para a realidade brasileira comparando os conhecimentos em matemática com expectativas quanto a disciplinas envolvendo estatística também são interessantes e salutares. E, para encerrar, pesquisas que não apenas diagnostiquem, mas também abordem novas formas de ensinar matemática e estatística nas escolas e nas universidades são necessárias para que o Brasil saia da posição desagradável em que se encontra no que se refere à aprendizagem dos alunos em comparação com diversos outros países.

\section{REFERÊNCIAS}

AUZMENDI, E. Las actitudes hacia la matemática-estadística en las enseñanzas medias y universitárias. Bilbao: Mensajero, 1992.

BAGOZZI, R.; YI, Y. On the evaluation of structural equation models. Journal of the Academy of Marketing Science, New York, v. 18, n. 1, p. 74-94, 1988.

BALOGLU, M. Individual differences in statistics anxiety among college students. Personality and Individual Differences, Amsterdam, v. 34, p. 855-865, 2003.

BALOGLU, M.; ZELHART, P. Statistical anxiety: A detailed review. Psychology and Education, Amstedam, v. 40, p. 27-37, 2003.

BANDURA, A. Self-efficacy. In RAMACHAUDRAN, V. (Org.). Encyclopaedia of human behavior. New York: Academic Press, 1994.

BAYOT, A.; MONDÉJAR, J.; MONDÉJAR, J.; MONSALVE, F.; VARGAS-VARGAS, M. The difficulties of learning concepts in the social sciences. In MISZTAL, M; TRAWINSKI, M. (Orgs.). Studies in teacher education: Psychopedagogy. Krakow: Wydawnictwo Naukowe Akademii Pedagogicznej, 2005.

BELL, J. Statistics anxiety and business statistics: The international student. Education, [s.1.], v. 129, n. 2, p. 282-286, 2008.

BRISLIN, R. Comparative research methodology: Cross-cultural studies. International Journal of Psychology, Abingdon, v. 11, n. 3, p. 215-229, 1976. 
CHEN, G.; GULLY, S.; EDEN, D. Validation of a new general self-efficacy scale. Organizational Research Methods, Thousand Oaks, v. 4, n. 1, p. 62-83, 2001.

CHIOU, C.; WANG, Y.; LEE, L. Reducing statistics anxiety and enhancing statistics learning achievement: Effectiveness of a one-minute strategy. Psychological Reports: Sociocultural Issues in Psychology, Thousand Oaks, v. 115, n. 1, p. 297-310, 2014.

CRUISE, R.; CASH, R.; BOLTON, D. Development and validation of an instrument to measure statistics anxiety. Proceedings of the American Statistical Association, Alexandria, p. 92-96, 1985.

DOLINSKY, B. An active learning approach to teaching statistics. Teaching of Psychology, Thousand Oaks, v. 28, n. 1, p. 55-56, 2001.

DOUGLAS, S.; CRAIG, C. Collaborative and iterative translation: an alternative approach to back translation. Journal of International Marketing, Chicago, v. 15, n. 1, p. 30-43, 2007.

EASLEY, R.; MADDEN, C.; DUNN, M. Conducting Marketing Science: The Role of Replication in the Research Process. Journal of Business Research, Amsterdam, v. 48, n. 1, p. 83-92, 2000.

ELMORE, P.; LEWIS, E.; BAY, M. Statistics achievement: a function of attitudes and related experience. In: Annual Meeting of the American Educational Research Association, april, 1993, Atlanta, GA. Proceedings... AERA: Atlanta, 1993.

EPSTEIN, I. Pedagogy of the perturbed: Teaching research to the reluctants. Journal of Teaching in Social Work, Abingdon, v. 1, n. 1, p. 71-89, 1987.

FORNELL, C.; LARCKER, D. Evaluating structural equations models with unobservable variables and measurement error. Journal of Marketing Research, Chicago, v. 18, n. 1, p. 39$50,1981$.

FORTE, J. Teaching statistics without sadistics. Journal of Social Work Education, Abingdon, v. 31, n. 2, p. 204-218, 1995.

HAIR, J.; HULT, G.; RINGLE, T.; SARSTEDT, M. A Primer on Partial Least Squares Structural Equation Modeling (PLS-SEM). Thousand Oaks: SAGE Publications, 2014. 
HE, J.; FREEMAN, L. Are Men More Technology-Oriented Than Women? The Role of Gender on the Development of General Computer Self-Efficacy of College Students. Journal of Information Systems Education, [s.1.], v. 21, n. 2, 203-212, 2010.

INEP. Disponível em: <http://portal.inep.gov.br/pisa-programa-internacional-de-avaliacaode-alunos>. Acesso em: 10 outubro. 2014.

JUNIUS, P.; SIDELL, N. Dispelling Fear and Loathing: Engaging Mathematically Challenged Students to Learn Statistics. The Journal of Baccalaureate Social Work, Alexandria,v. 14, n. 2, p. 49-61, 2009.

LALAYANTS, M. Overcoming Graduate Students' Negative Perceptions of Statistics. Journal of Teaching in Social Work, Abingdon, v. 32, n. 4, p. 356-375, 2012.

LALONDE, R.; GARDNER, R. Statistics as a second language? A model for predicting performance in psychology students. Canadian Journal of Behavioral Science, Washington,v. 25, p. 108-125, 1993.

MACKAY, J.; PARKINSON, J. Gender, self-efficacy and achievement among South African Technology teacher trainees. Gender and Education, Abingdon, v. 22, n. 1, p. 87-103, 2010.

MALHOTRA, N. Marketing research: An applied orientation. New Jersey: Prentice Hall, 1999.

MONDÉJAR-JIMÉNEZ, J.; VARGAS-VARGAS, M. Determinant factors of attitude towards quantitative subjects: Differences between sexes. Teaching and Teacher Education, Amsterdam, v. 26, n. 3, p. 688-693, 2010.

ONWUEGBUZIE, A. The interaction of statistics test anxiety and examination condition in statistics achievement of post-graduate non-statistics majors. 1993. Tese (Doutorado) University of South Carolina.

ONWUEGBUZIE, A.; SEAMAN, M. The effect of time and anxiety on statistics achievement. Journal of Experimental Psychology, Washington, v. 63, p. 115-12, 1995.

ONWUEGBUZIE, A. Writing a research proposal: the role of library anxiety, statistics anxiety, and composition anxiety. Library \& Information Science Research, Amsterdam, v. 19, n. 1, p. 5-33, 1997. 
ONWUEGBUZIE, A. Attitudes toward statistics assessments. Assessment \& Evaluation in Higher Education, Abingdon, v. 25, n. 4, p. 321-339, 2000.

ONWUEGBUZIE, A.; SLATE, J.; PATERSON, F. WATSON; M.; SCHWARTZ, R. Factors associated with achievement in educational research courses. Research in Schools, [s.1.], v. 7, n. 1, p. 53-65, 2000.

ONWUEGBUZIE, A. Modeling statistics achievement among graduate students. Educational and Psychological Measurement, Thousand Oaks, v. 63, n. 6, p. 1020-1038, 2003.

ONWUEGBUZIE, A.; WILSON, V. Statistics anxiety: Nature, etiology, antecedents, effects, and treatments: A comprehensive review of the literature. Teaching in Higher Education, Abingdon, v. 8, n. 2, p. 195-209, 2003.

ONWUEGBUZIE, A. Academic procrastination and statistics anxiety. Assessment \& Evaluation in Higher Education, Abingdon, v. 29, n. 1, p. 3-19, 2004.

PAN, W.; TANG, M. Students' perceptions on factors of statistics anxiety and instructional strategies. Journal of Instructional Psychology, [s.1.], v. 32, n. 3, p. 205-214, 2004.

SCHACHT, S.; STEWART, B. What's funny about statistics? A technique for reducing student anxiety. Teaching Sociology, Thousand Oaks, v. 18, n. 1, p. 52-56, 1990.

SRITE, M.; KARAHANNA, E. The role of espoused national cultural values in technology acceptance. MIS Quarterly, Mineapolis, v. 30, n. 3, p. 679-704, 2006.

UTTL, B.; WHITE, C.; MORIN, AL. The Numbers Tell It All: Students Don't Like Numbers! PLos One, San Francisco, v. 8, n. 12, p. 1-9, 2013.

ZEIDNER, M. Statistics and mathematics anxiety in social science students - some interesting parallels. British Journal of Educational Psychology, Hoboken, v. 61, n. 3, p. 319-328, 1991.

ZHANG, Y.; SHANG, L.; WANG, R.; ZHAO, Q.; LI, C.; XU, Y.; SU, H. Attitudes toward statistics in medical postgraduates: measuring, evaluating and monitoring. Medical Education, Hoboken, v. 12, n. 117, p. 1-8, 2012. 